\title{
Successful Treatment of Spine Fracture for Diffuse Idiopathic Skeletal Hyperostosis with Teriparatide-A Report of Two Cases
}

\author{
Yasuaki Iida, Hiroshi Takahashi", Yuichiro Yokoyama, Yasuhiro Inoue, Daisuke Suzuki, \\ Keiji Hasegawa, Shintaro Tsuge, Wataru Shishikura, Katsunori Fukutake, Ryo Takamatsu, \\ Kazumasa Nakamura, Masayuki Sekiguchi, Akihito Wada
}

Department of Orthopaedic Surgery, Toho University School of Medicine, Tokyo, Japan.

Email: "drkan@med.toho-u.ac.jp

Received August 29 $9^{\text {th }}, 2013$; revised September $28^{\text {th }}, 2013$; accepted October $6^{\text {th }}, 2013$

Copyright (C) 2013 Yasuaki Iida et al. This is an open access article distributed under the Creative Commons Attribution License, which permits unrestricted use, distribution, and reproduction in any medium, provided the original work is properly cited.

\begin{abstract}
We experienced 2 cases of spinal fracture in patients aged 80 years or older with diffuse idiopathic skeletal hyperostosis (DISH). Since they decided not to undergo surgical treatment, we provided conservative treatment with teriparatide. There has been no previous study on teriparatide for spine fracture in DISH. This is the first report of the successful use of teriparatide to treat spine fracture in DISH without surgical intervention. Our patients were treated with teriparatide for 8 - 9 months after diagnosis of the fracture. Union was obtained in two cases and no adverse events were observed during treatment. Our report showed that teriparatide could be an alternative to conventional intervention in spinal fracture of DISH.
\end{abstract}

Keywords: Diffuse Idiopathic Skeletal Hyperostosis; Teriparatide; Spine Fracture

\section{Introduction}

Patients with diffuse idiopathic skeletal hyperostosis (DISH) may experience problems in that spinal bone fractures can occur in response to slight external forces. In such bone fractures, it is difficult to ensure that bone union is achieved with conservative treatment, as seen in transverse fracture of a long bone.

Since such bone fractures occur as low-energy trauma, there is a potential that the bone fracture could be overlooked at the first hospital visit and diagnosed as a dislocation, and that inappropriate treatment would then be provided.

For bone fractures that occur in patients with ankylosing spinal disorders (ASD), such as ankylosing spondylitis (AS) and DISH, surgical treatment is recommended rather than conservative treatment, based on the results of analyses of the incidence of complications and prognosis [1].

However, it has also been reported that the incidence of postoperative complications and the mortality would

\footnotetext{
"Conflict of interest: None.

Financial support: None.

${ }^{\#}$ Corresponding author.
}

be high because DISH patients tend to be older in many cases $[1,2]$.

Herein, we report our experience of 2 cases, in which bone union was achieved by using conservative treatment with teriparatide, thus, enabling walking with no lumbar pain. For these cases, we did not select surgical treatment because the patients were among extreme old age and had various medical comorbidities.

\section{Case 1}

\section{An 83-Year-Old Female Patient}

The patient had a history of type II diabetes, cerebral infarction and cardiac infarction, and she had received insulin therapy for diabetes, and stent placement in the anterior descending branch for cardiac infarction.

She visited our hospital for persistent lumbar pain, which began after she stumbled and fell down in a room.

She had percussion tenderness at the spinous process in the thoracolumbar spine, and the pain was increased by body motion.

Although plain X-ray findings suggested DISH, no clear bone fracture was confirmed (Figure 1). 


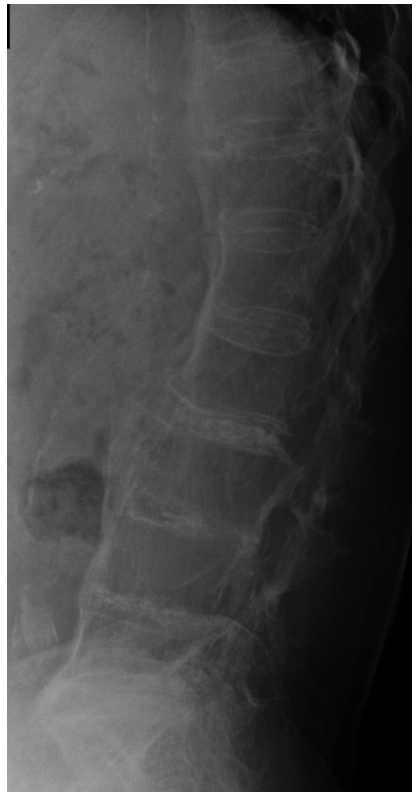

Figure 1. Lateral plain $\mathrm{X}$-ray image at the first hospital visit. Ossification of the anterior longitudinal ligament was confirmed in a wide area from the thoracic vertebrae to the lumbar vertebrae.

MRI showed a fracture line passing from the ossified area of the anterior longitudinal ligament at Th12/L1 level through the inferior border of the Th12 vertebral body. Based on these findings, the patient was diagnosed as having a bone fracture of DISH caused by a slight external force (Figure 2).

The patient was hospitalized for complete rest. Although surgical treatment was considered for severe pain in the dorsal position, we began to provide conservative treatment with teriparatide because the patient and her family did not wish her to undergo surgical treatment due to her extreme old age and the high risk of various medical comorbidities.

One month after commencement of conservative treatment, she became aware of improvement in the lumbar pain, and after 2 months, she was able to walk using a corset and a T-cane.

In CT findings obtained 4 months after development of the bone fracture, formation of a callus was confirmed, although local kyphosis was observed at the fracture site, and the patient could walk for about 5 minutes using a T-cane. X-ray and CT findings obtained 6 months after the development of bone fracture showed favorable formation of the callus, and she was aware of no lumbar pain and was able to climb and descend stairs (Figure 3).

\section{Case 2}

\section{An 83-Year-Old Male Patient}

The patient had a history of type II diabetes, Chronic

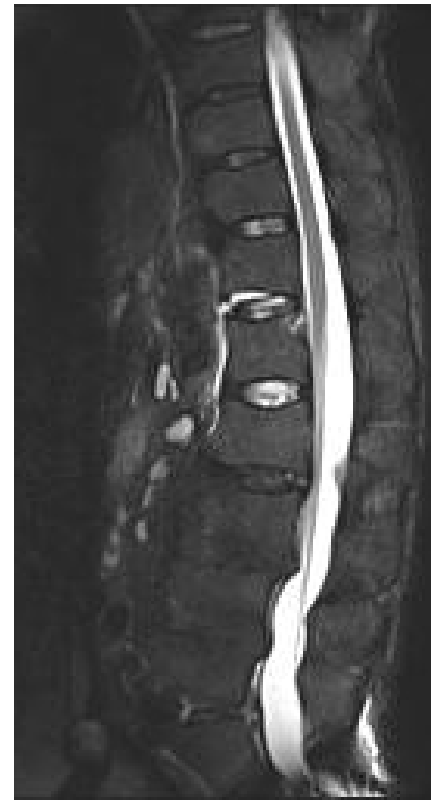

Figure 2. Sagittal STIR image of lumbar spine MRI. T1weighted image shows a linear low intensity area along the end plate on the Th12 caudal vertebral body, whereas the T2-weighted image shows a linear high intensity area. In the STIR image, a high intensity area was confirmed from the area along the anterior border of the caudal vertebral body.

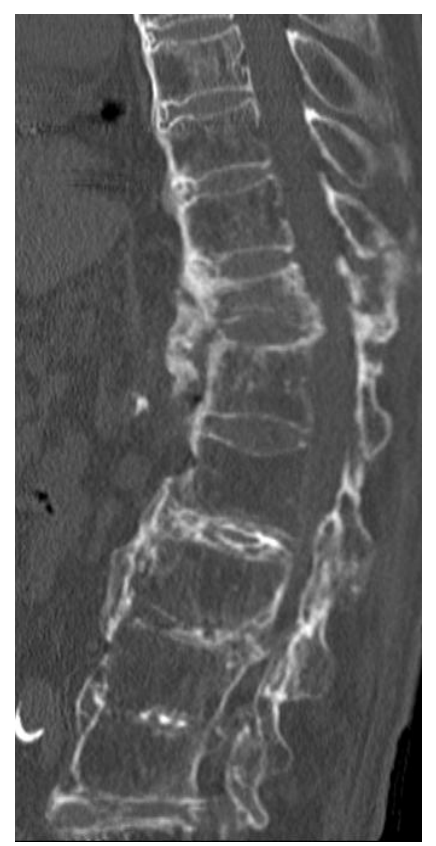

Figure 3. Sagittal CT image. Although local kyphosis was observed in the fracture site, 6 months after bone fracture favorable formation of callus was confirmed.

Obstructive Pulmonary Disease (COPD), and cerebral infarction. He had received insulin therapy for diabetes and domiciliary oxygen therapy for COPD. 
When he left home, he lost his balance and fell down. He subsequently developed lumbar pain and difficulty in walking, and was referred to our hospital.

When he visited our hospital, he had percussion tenderness at the spinous process of the lower lumbar spine. Based on plain X-ray finings, he was diagnosed as having DISH, and compressed fracture of the L3, L4 vertebral body in the discontinuous ossified site of the anterior longitudinal ligament was suspected (Figure 4).

MRI showed bone fracture of the L3, L4 vertebral body (Figure 5).

He was hospitalized for complete bed rest, and surgical treatment was considered due to the severe pain in the dorsal position. After consultation with the patient and his family regarding therapeutic selection, it was decided to begin treatment with teriparatide since they did not wish him to undergo surgical treatment due to the extreme old age and the high risk of medical comorbidities.

One month after commencement of the treatment with teriparatide, the lumbar pain improved, and after 2 months, the patient was able to walk using a T-cane.

Plain X-ray images taken 6 months after the development of the bone fracture showed clear crush of the L3, L4 vertebral body, and formation of a callus in the anterior longitudinal ligament. Flexion-extension radiograph

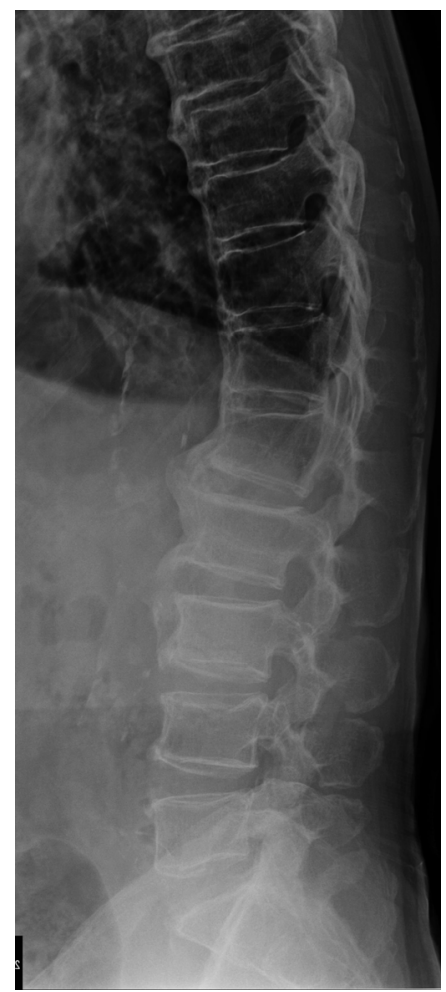

Figure 4. Lateral plain $X$-ray image obtained at the first hospital visit. The irregular end plate was confirmed on the inferior border of the $L 3$ vertebral body and the superior border of the $L 4$ vertebral body.

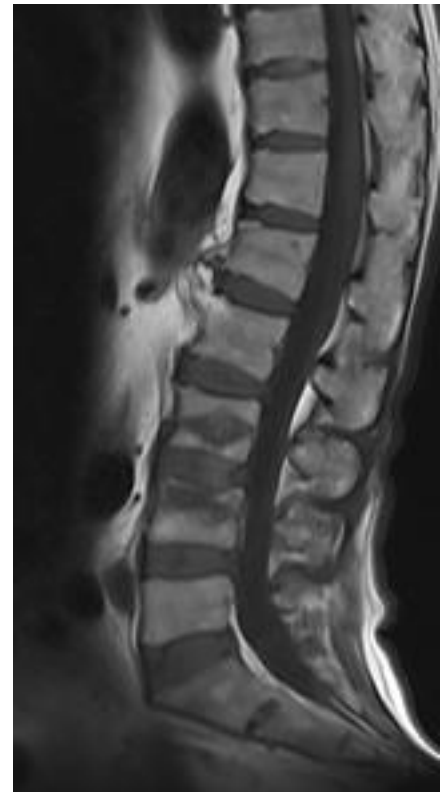

Figure 5. T1-weighted MR image at the first hospital visit. The bone fracture was confirmed with a low intensity area in the T1-weighted image for the L3, L4 vertebral body.

showed no abnormal mobility at the site, and the lumbar pain was cured, enabling him to be engaged in daily living, as he had before development of the bone fracture (Figure 6).

\section{Discussion}

DISH is a supposedly non-inflammatory disease in which the spinal longitudinal ligaments and entheses slowly become ossified. While the clinical entity of DISH was first described by Resnick et al. [3], its etiology remains unknown. However, there have been reports of associations with obesity, type 2 diabetes mellitus and advanced age $[4,5]$.

According to Westerveld et al., surgical treatment is recommended for bone fracture in patients with ASD including DISH, rather than conservative treatment, based on the incidence of complications and prognosis [1].

Caron et al. [2] also suggested that the mortality of the surgically-treated group was $23 \%$, compared to $51 \%$ mortality with no operative treatment, and the mortality was significantly lower in patients aged 70 years or older.

Teriparatide is a recombinant bioactive fragment (1-34) of human parathyroid hormone and is indicated for the treatment of postmenopausal osteoporosis. It has been shown to stimulate osteoblasts, enhance bone connectivity, increase endosteal cortical thickness, and improve bone mineral content [6-10]. The efficacy of teriparatide on human osteoporosis has already been established, and many reports have suggested its preventive and analgesic 


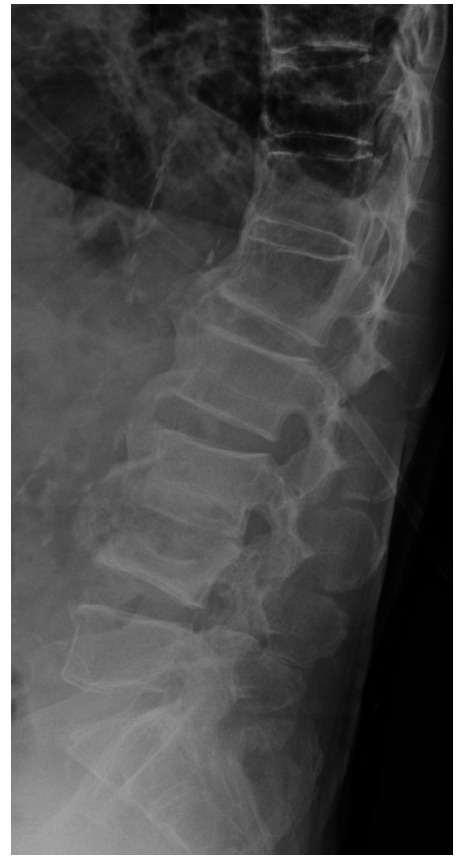

Figure 6. Plain $\mathrm{X}$-ray findings obtained 6 months after bone fracture. L3, L4 vertebral body was crushed, but formation of a callus was confirmed in the anterior longitudinal ligament.

effects for osteoporosis-derived spinal compression fracture [11-13]. In addition, several recent reports have suggested that teriparatide would exhibit efficacy to promote healing of bone fracture and pseudoarthrosis by aggressively stimulating osteoblasts to promote bone formation [14-17].

For our 2 cases, we had no choice but to select conservative treatment because of the high risk of surgical treatment and the wishes of the patients and their families.

To date, there have been no reports on the use of teriparatide in the treatment of spinal fracture associated with DISH. However, we used the teriparatide to treat our cases based on the reports of the usefulness for pseudoarthrosis at various sites and the promotion of healing of bone fracture. As a result, both of the patients experienced improved pain at the fracture site at a comparatively early phase, and active formation of callus was confirmed in CT images 4 months after the bone fracture in one case. Six months after bone fracture, both of the patients had no lumbar pain and were able to engage in normal daily activities, as they had before the bone fracture.

For bone fracture in patients with ASD, surgical treatment is recommended, rather than conservative treatment. However, the incidence of postoperative complications can be high in patients who undergo surgical treatment. Teriparatide could be a medicine that will fundamentally change the concept for conventional treatment methods.

\section{REFERENCES}

[1] L. A. Westerveld, J. J. Verlaan and F. C. Oner, "Spinal Fractures in Patients with Ankylosing Spinal Disorders: A Systematic Review of the Literature on Treatment, Neurological Status and Complications,” European Spine Journal, Vol. 18, 2009, pp. 145-156.

http://dx.doi.org/10.1007/s00586-008-0764-0

[2] T. Caron, R. Bransford, Q. Nguyen, J. Agel, J. Chapman and C. Bellabarba, "Spine Farctures in Patients with Ankylosisng Spinal Disorders,” Spine, Vol. 35, 2010, pp. E458-E464.

[3] D. Resnick and G. Niwayama, "Radiographic and Pathologic Features of Spinal Involvement in Diffuse Idiopathic Skeletal Hyperostosis,” Radiology, Vol. 119, 1976, pp. 559-568.

[4] C. W. Denko and C. J. Malemud, "Body Mass Index and Blood Glucose: Correlations with Serum Insulin, Growth Hormone, and Insulin-Like Growth Factor-1 Levels in Patients with Diffuse Idiopathic Skeletal Hyperostosis (DISH),” Rheumatology International, Vol. 26, 2006, pp. 292-297. http://dx.doi.org/10.1007/s00296-005-0588-8

[5] C. Kiss, M. Szilagyi, A. Paksy and G. Poor, "Risk Factors for Diffuse Idiopathic Skeletal Hyperostosis: A CaseControl Study,” Rheumatology, Vol. 41, 2002, pp. 27-30. http://dx.doi.org/10.1093/rheumatology/41.1.27

[6] E. Canalis, A. Giustina and J. P. Bilezikian, "Mechanisms of Anabolic Therapies for Osteoporosis,” The New England Journal of Medicine, Vol. 357, 2007, pp. 905-916. http://dx.doi.org/10.1056/NEJMra067395

[7] M. Gass and B. Dawson-Hughes, "Preventing Osteoporosis-Related Fractures: An Overview,” American Journal of Medicine, Vol. 119, 2006, pp. 3S-11S. http://dx.doi.org/10.1016/j.amjmed.2005.12.017

[8] R. P. Heaney, “Advances in Therapy for Osteoporosis," Clinical Medicine \& Research, Vol. 1, 2003, pp. 93-99. http://dx.doi.org/10.3121/cmr.1.2.93

[9] Y. Jiang, J. J. Zhao, B. H. Mitlak, O. Wang, H. K. Genant and E. F. Eriksen, "Recombinant Human Parathyroid Hormone(1-34)(Teriparatide) Improves Both Cortical and Cancellous Bone Structure,” Journal of Bone and Mineral Research, Vol. 18, 2003, pp. 1932-1941. http://dx.doi.org/10.1359/jbmr.2003.18.11.1932

[10] R. Lindsay, H. Zhou, F. Cosman, J. Nieves, D. W. Dempster and A. B. Hodsman, "Effects of a One-Month Treatment with pth(1-34) on Bone Formation on Cancellous, Endocortical, and Periosteal Surfaces of the Human Ilium," Journal of Bone and Mineral Research, Vol. 22, 2007, pp. 495-502. http://dx.doi.org/10.1359/jbmr.070104

[11] R. M. Neer, C. D. Arnaud, J. R. Zanchetta, R. Prince, G. A. Gaich, J. Y. Reginster, A. B. Hodsman, E. F. Eriksen, S. Ish-Shalom, H. K. Genant, O. Wang and B. H. Miltlak, "Effect of Parathyroid Hormone(1-34) on Fractures and Bone Mineral Density in Postmenopausal Women with Osteoporosis," The New England Journal of Medicine, Vol. 10, 2001, pp. 1434-1441. 
http://dx.doi.org/10.1359/jbmr.070104

[12] F. M. Ulivieri, "Back Pain Treatment in Post-Menopausal Osteoporosis with Nertebral Fractures,” Aging Clinical and Experimental Research, Vol. 19, 2007, pp. 21-23.

[13] C. H. Su, P. H. Tu, T. C. Yang and Y. Y. Tseng, "Comparison of the Therapeutic Effect of Teriparatide with That of Combined Vertebroplasty with Antiresorptive Agents for the Treatment of New-Onset Adjacent Vertebral Compression Fracture after Percutaneous Vertebroplasty," Journal of Spinal Disorders \& Techniques, Vol. 26, 2013, pp. 200-206. http://dx.doi.org/10.1097/BSD.0b013e31823f6298

[14] P. T. Rubery and S. V. Bukata, “Teriparatide May Acceerate Healing in Delayed Unions of Type III Odontoid Fractures. A Report of 3 Cases,” Journal of Spinal Disorders \& Techniques, Vol. 23, 2010, pp. 151-155.
http://dx.doi.org/10.1097/BSD.0b013e31819a8b7a

[15] Y. K. Lee, Y. C. Ha and K. H. Koo, "Teriparatide, a Nonsurgical Solution for Femoral Nonunion? A Report of Three Cases,” Osteoporosis International, Vol. 23, 2012, pp. 2897-2900.

http://dx.doi.org/10.1097/BSD.0b013e31819a8b7a

[16] S. Chintamaneni, K. Finzel and B. L. Gruber, "Successful Treatment of Sternal Fracture Nonunion with Teriparatide,” Osteoporosis International, Vol. 21, 2010, pp. 1059-1063. http://dx.doi.org/10.1007/s00198-009-1061-4

[17] A. P. Johansson, “Teriparatide Improves Early Callus Formation in Distal Radial Fractures. Analysis of a Sub Group of Patients within a Randomized Trial," Acta Orthopaedica, Vol. 81, 2010, pp. 234-236.

http://dx.doi.org/10.3109/17453671003761946 